\title{
Pseudodifferential equations, wave factorization, and related problems
}

\author{
Vladimir B. Vasilyev (1)
}

Department of Differential Equations, Belgorod State National Research

University, Belgorod, Russia

\section{Correspondence}

Vladimir B. Vasilyev, Chair of Differential Equations, Department of Differential Equations, Belgorod State National Research University, Studencheskaya 14/1, Belgorod 308007, Russia.

Email: vladimir.b.vasilyev@gmail.com

MSC Classification: $35 \mathrm{C} 05$; 47G30

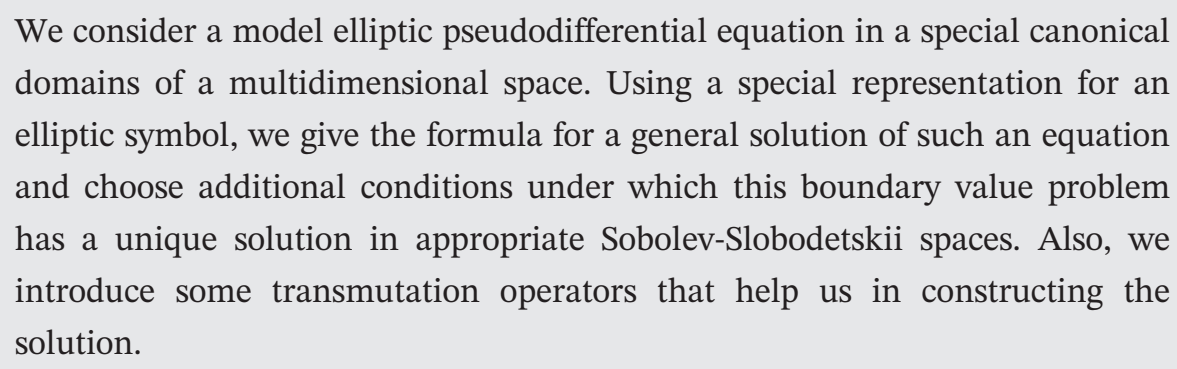

We consider a model elliptic pseudodifferential equation in a special canonical domains of a multidimensional space. Using a special representation for an elliptic symbol, we give the formula for a general solution of such an equation and choose additional conditions under which this boundary value problem has a unique solution in appropriate Sobolev-Slobodetskii spaces. Also, we introduce some transmutation operators that help us in constructing the solution.

\section{KEYWORDS}

boundary value problem, polyhedral angle, pseudodifferential equation, solvability, transmutation operator, wave factorization

\section{1 | INTRODUCTION}

The author develops the theory of boundary value problems based on two principles. ${ }^{1}$ These are a local principle and factorizability principle at a boundary point like. ${ }^{2-4}$ The first principle was known earlier, and it also was known as a freezing coefficients principle. Usually, the second name corresponds to partial differential equations theory, but the first name was introduced for multidimensional singular integral equations and, more general, for pseudodifferential equations. The last case permits to obtain rough properties for pseudodifferential equations and related boundary value problems, namely, Fredholm properties only in comparison with differential operators and boundary value problems where one has as a rule results on existence and uniqueness.

There are a lot of approaches to construct such a theory (see, for example, previous studies ${ }^{5-10}$ ). I have written many times $^{11,12}$ what is the difference between this consideration and others; it is a choice of distinct key principles. In any case, one needs to declare an invertibility of so-called local representatives of an initial pseudodifferential operator to describe its Fredholm properties.

Local principle and factorizability was first introduced in Simonenko ${ }^{4}$ (for multidimensional singular integral operators in Lebesgue $L_{p}$-spaces) and Eskin ${ }^{3}$ (for pseudodifferential operators in Sobolev-Slobodetskii $H^{s}$-spaces). For manifolds with a smooth boundary, the problem reduces to a half-space case, for which a factorizability principle holds immediately because under localization at a boundary point and applying the Fourier transform, we obtain a wellknown one-dimensional classical Riemann boundary value problem for upper and lower complex half planes with a multidimensional parameter. This approach does not work if a boundary has at least one singular point like a conical point. One needs here other considerations and approaches.

Such operators and equations appear in a lot of applied problems that simulate different real situations (see, for example, Cordero et $\mathrm{al}^{13}$ and Rodino et $\mathrm{al}^{14}$ ). Therefore, it is very important to find new approaches and methods for studying these mathematical objects. 


\section{2 | OPERATORS IN CONES}

Our main goal is to describe possible solvability conditions for the pseudodifferential equation

$$
(A u)(x)=f(x), x \in D,
$$

where $D$ is manifold with a boundary and $A$ is pseudodifferential operator with the symbol $A(x, \xi)$.

Such operators are defined locally by the formula

$$
u(x) \mapsto \int_{\mathbb{R}^{m}} \int_{\mathbb{R}^{m}} A(x, \xi) u(y) e^{-i(y-x) \cdot \xi} d \xi d y,
$$

... if $D$ is a smooth compact manifold because one can use freezing coefficients principle, or in other words local principle. For manifold with a smooth boundary, we need a new local formula for defining the operator $A$ : More precisely, in inner points of $D$, we use the formula (1), but in boundary points, we need another formula

$$
u(x) \mapsto \int_{\mathbb{R}_{+}^{m}} \int_{\mathbb{R}^{m}} A(x, \xi) u(y) e^{-i(y-x) \cdot \xi} d \xi d y
$$

For invertibility of such operator with symbol $A(\cdot, \xi)$ nondepending on spatial variable $x$, one can apply the theory of classical Riemann boundary problem for upper and lower complex half planes with a parameter $\xi^{\prime}$. This step was systematically studied in the book. ${ }^{3}$ But if the boundary $\partial D$ has at least one conical point, this approach is not effective.

The conical point at the boundary is such a point, for which its neighborhood is diffeomorphic to the cone $C \subset \mathbb{R}^{m}$; this cone $C$ should be a sharp convex cone nonincluding a whole straight line; hence, the local definition for pseudodifferential operator near the conical point is the following

$$
u(x) \mapsto \int_{C} \int_{\mathbb{R}^{m}} A(x, \xi) u(y) e^{-i(y-x) \cdot \xi} d \xi d y
$$

We consider the operator (2) in the Sobolev-Slobodetskii space $H^{s}\left(\mathbb{R}^{m}\right)$ with norm

$$
\|u\|_{s}^{2}=\int_{\mathbb{R}^{m}}|\tilde{u}(\xi)|^{2}(1+|\xi|)^{2 s} d \xi
$$

and introduce the following class of symbols nondepending on spatial variable $x$ : $\exists c_{1}, c_{2}>0$, such that

$$
c_{1} \leq\left|A(\xi)(1+|\xi|)^{-\alpha}\right| \leq c_{2}, \xi \in \mathbb{R}^{m} .
$$

The number $\alpha \in \mathbb{R}$ we call the order of pseudodifferential operator $A$.

It is well known that pseudodifferential operator with symbol $A(\xi)$ satisfying (2) is a linear bounded operator acting from $H^{s}\left(\mathbb{R}^{m}\right)$ into $H^{s-\alpha}\left(\mathbb{R}^{m}\right) .^{3}$

We are interested in studying invertibility operator (2) in corresponding Sobolev-Slobodetskii spaces. By definition, $H^{s}(C)$ consists of distributions from $H^{s}\left(\mathbf{R}^{m}\right)$ with support in $\bar{C}$. The norm in the space $H^{S}(C)$ is induced by the norm $H^{S}\left(\mathbb{R}^{m}\right)$. We associate such operator with corresponding equation

$$
(A u)(x)=f(x), x \in C,
$$

where right-hand side $f$ is chosen from the space $H_{0}^{s-\alpha}(C)$.

$H_{0}^{s}(C)$ is the space of distributions $S^{\prime}(C)$, which admit continuation on $H^{s}\left(\mathbf{R}^{m}\right)$. The norm in $H_{0}^{s}(C)$ is defined by

$$
\|f\|_{s}^{+}=\inf \|l f\|_{s}
$$

where infimum is chosen for all possible continuations $l$. 


\section{3 | THE WAVE FACTORIZATION}

Below, we will consider the symbols $A(\xi)$ satisfying the condition (3).

Let us denote by $\stackrel{*}{C}$ the conjugate cone

$$
\stackrel{*}{C}=x \in \mathbf{R}^{m}:\{x \cdot y>0, \forall y \in C\} .
$$

Definition 1. Wave factorization of symbol $A(\xi)$ with respect to the cone $C$ is called its representation in the form

$$
A(\xi)=A_{\neq}(\xi) A_{=}(\xi)
$$

where the factors $A_{\neq}(\xi), A_{=}(\xi)$ satisfy the following conditions:

1. $A_{\neq}(\xi), A_{=}(\xi)$ are defined everywhere without may be the points $\left\{\xi \in \mathbb{R}^{m}: \xi \in \partial(\stackrel{*}{C} \cup(-\stackrel{*}{C}))\right\}$;

2. $A_{\neq}(\xi), A_{=}(\xi)$ admit an analytical continuation into radial tube domains $T(\stackrel{*}{C}), T(-\stackrel{*}{C})$, respectively, which satisfy the estimates

$$
\begin{aligned}
& \left|A_{\neq}^{ \pm 1}(\xi+i \tau)\right| \leq c_{1}(1+|\xi|+|\tau|)^{ \pm \infty}, \\
& \left|A_{\equiv}^{ \pm 1}(\xi-i \tau)\right| \leq c_{2}(1+|\xi|+|\tau|)^{ \pm(\alpha-\infty)}, \forall \tau \in C^{*} .
\end{aligned}
$$

The number $æ$ is called index of wave factorization.

Example 1. Let $C \equiv C_{+}^{a}=\left\{x \in \mathbb{R}^{m}: x_{m}>a\left|x^{\prime}\right|, x^{\prime}=\left(x_{1}, \cdots, x_{m-1}\right), a>0\right\}$,

$$
A=-\frac{\partial^{2}}{\partial x_{1}^{2}}-\cdots-\frac{\partial^{2}}{\partial x_{m}^{2}}+k^{2}, \quad k \in \mathbb{R} \backslash\{0\},
$$

and then according to some properties of the Fourier transform, the symbol of this operator has the form

$$
A(\xi)=\xi_{1}^{2}+\xi_{2}^{2}+\cdots+\xi_{m}^{2}+k^{2} .
$$

The following equality is the wave factorization of the Helmholtz operator. We will write it as

$$
\begin{aligned}
& \xi_{m}^{2}+\left|\xi^{\prime}\right|^{2}+k^{2}= \\
& \left(\sqrt{a^{2}+1} \xi_{m}+\sqrt{a^{2} \xi_{m}^{2}-\left|\xi^{\prime}\right|^{2}-k^{2}}\right)\left(\sqrt{a^{2}+1} \xi_{m}-\sqrt{a^{2} \xi_{m}^{2}-\left|\xi^{\prime}\right|^{2}-k^{2}}\right)
\end{aligned}
$$

meaning for $\sqrt{a^{2} \xi_{m}^{2}-\left|\xi^{\prime}\right|^{2}-k^{2}}$ the boundary value

$$
\sqrt{a^{2}\left(\xi_{m}+i 0\right)^{2}-\left|\xi^{\prime}\right|^{2}-k^{2}}
$$

\section{I PSEUDODIFFERENTIAL EQUATIONS, TRANSMUTATION OPERATORS, AND SOLVABILITY}

Here, we will consider a special cone of the kind

$$
C_{+}^{a}=\left\{x \in \mathbf{R}^{m}: x_{m}>\sum_{k=1}^{m-1} a_{k}\left|x_{k}\right|, a_{k}>0\right\}
$$

and Equation 4 for the case $æ-s=n+\delta, n \in \mathbf{N},|\delta|<1 / 2$, only. 
Let $T$ be the bijection operator transferring $\partial C_{+}^{a}$ into hyperplane $x_{m}=0$; more precisely, it is transformation $\mathbf{R}^{m} \rightarrow \mathbf{R}^{m}$ of the following type

$$
\left\{\begin{array}{c}
t_{1}=x_{1}, \\
\cdots \cdots \cdots \cdots \\
t_{m-1}=x_{m-1}, \\
t_{m}=x_{m}-\sum_{k=1}^{m-1} a_{k}\left|x_{k}\right|,
\end{array}\right.
$$

and we introduce the operator

$$
F T F^{-1} \equiv V_{a}
$$

where $\mathbf{a}=\left(a_{1}, a_{2}, \ldots, a_{m-1}\right)$ and, further, one can construct the general solution for our pseudodifferential Equation 4.

Theorem 1. A general solution of the Equation 4 in Fourier image is given by the formula

$$
\begin{aligned}
\tilde{u}(\xi)= & A_{\neq}^{-1}(\xi) Q(\xi) G_{m} Q^{-1}(\xi) A_{=}^{-1}(\xi) \tilde{l} \tilde{f}(\xi)+ \\
& +A_{\neq}^{-1}(\xi) V_{-a} F\left(\sum_{k=1}^{n} c_{k}\left(x^{\prime}\right) \delta^{(k-1)}\left(x_{m}\right)\right),
\end{aligned}
$$

where $c_{k}\left(x^{\prime}\right) \in H^{s_{k}}\left(\mathbf{R}^{m-1}\right)$ are arbitrary functions, $\mathrm{s}_{\mathrm{k}}=\mathrm{s}-\alpha+\mathrm{k}-1 / 2, \mathrm{k}=1,2, \ldots, \mathrm{n}$, if is an arbitrary continuation $\mathrm{f}$ on $\mathrm{H}^{\mathrm{s}-\alpha}\left(\boldsymbol{R}^{\mathrm{m}}\right)$, and $\mathrm{Q}(\xi)$ is an arbitrary polynomial satisfying (3) for $\alpha=\mathrm{n}$. The a priori estimate holds

$$
\|u\|_{s} \leq C\left(\|f\|_{s-\alpha}^{+}+\left[c_{k}\right]_{s_{k}}\right),
$$

where $[\cdot]_{s_{k}}$ denotes $H^{s_{k}}\left(\mathbf{R}^{m-1}\right)$-norm.

Proof We continue $f$ on whole $\mathbf{R}^{m}$ and denote this continuation by $l f$. Then we put

$$
u_{-(x)}=(l f)(x)-(A u)(x),
$$

so that $u_{-}(x)=0$ in the cone $C_{+}^{a}$. The general solution is constructed by the following way. After wave factorization for symbol with preliminary Fourier transform, we write

$$
A_{\neq}(\xi) \tilde{u}(\xi)+A_{=}^{-1}(\xi) \tilde{u}_{-}(\xi)=A_{=}^{-1}(\xi) \tilde{l} \tilde{f}(\xi) .
$$

One can see that $A_{=}^{-1}(\xi) \tilde{l} \tilde{f}(\xi)$ belongs to the space $\tilde{H}^{s-a}\left(\mathbf{R}^{m}\right)$, and if we choose the polynomial $Q(\xi)$, satisfying the condition

$$
|Q(\xi)| \sim(1+|\xi|)^{n}
$$

then $Q^{-1}(\xi) A_{=}^{-1}(\xi) \tilde{l}(\xi)$ belongs to the space $\tilde{H}^{-\delta}\left(\mathbf{R}^{m}\right)$. Further, according to the theory of multidimensional Riemann problem, ${ }^{1}$ we can decompose the last function on two summands (jump problem):

$$
Q^{-1} A_{=}^{-1} \tilde{l f}=f_{+}+f_{-},
$$

where $f_{+} \in \tilde{H}\left(C_{+}^{a}\right), f_{-} \in \tilde{H}\left(\mathbf{R}^{m} \backslash C_{+}^{a}\right)$ and

$$
f_{+}=G_{m} Q^{-1} A_{=}^{-1} \tilde{l f} .
$$

So we have

$$
Q^{-1} A_{\neq} \tilde{u}+Q^{-1} A_{=}^{-1} \tilde{u}_{-}=f_{+}+f_{-},
$$

or

$$
Q^{-1} A_{\neq} \tilde{u}-f_{+}=f_{-}-Q^{-1} A_{=}^{-1} \tilde{u}_{-} .
$$


In other words,

$$
A_{\neq} \tilde{u}-Q f_{+}=Q f_{-}-A_{=}^{-1} \tilde{u}_{-} .
$$

The left-hand side of the equality belongs to the space $\tilde{H}^{-n-\delta}\left(C_{+}^{a}\right)$, and the right hand belongs to $\tilde{H}^{-n-\delta}\left(\mathbf{R}^{m} \backslash C_{+}^{a}\right)$; hence,

$$
F^{-1}\left(A_{\neq} \tilde{u}-Q f_{+}\right)=F^{-1}\left(Q f_{-}-A_{=}^{-1} \tilde{u}_{-}\right),
$$

where the left-hand side belongs to $H^{-n-\delta}\left(C_{+}^{a}\right)$ and the right-hand side belongs to $H^{-n-\delta}\left(\mathbf{R}^{m} \backslash C_{+}^{a}\right)$, from which we conclude immediately, it is distribution supported on $\partial C_{+}^{a}$.Then the function

$$
T F^{-1}\left(A_{\neq} \tilde{u}-Q f_{+}\right)
$$

is supported on the hyperplane $t_{m}=0$ and belongs to $H^{-n-\delta}\left(\mathbf{R}^{m}\right)$. Such distribution belongs to the subspace generated by a Dirac mass function and its derivatives, ${ }^{15}$ and it looks the following:

$$
\sum_{k=0}^{n-1} c_{k}\left(t^{\prime}\right) \delta^{(k)}\left(t_{m}\right)
$$

Therefore,

$$
T F^{-1}\left(A_{\neq} \tilde{u}-Q f_{+}\right)=\sum_{k=0}^{n-1} c_{k}\left(t^{\prime}\right) \delta^{(k)}\left(t_{m}\right) .
$$

Further, we apply the Fourier transform

$$
\operatorname{FTF}^{-1}\left(A_{\neq} \tilde{u}-Q f_{+}\right)=F\left(\sum_{k=0}^{n-1} c_{k}\left(t^{\prime}\right) \delta^{(k)}\left(t_{m}\right)\right),
$$

taking into account (5) and obtain

$$
A_{\neq}(\xi) \tilde{u}(\xi)-Q(\xi) f_{+}(\xi)=V_{-a} F\left(\sum_{k=0}^{n-1} c_{k}\left(t^{\prime}\right) \delta^{(k)}\left(t_{m}\right)\right)
$$

from which according to (6), we have

$$
\begin{gathered}
\tilde{u}(\xi) \quad=A_{\neq}^{-1}(\xi) Q(\xi) G_{m} Q^{-1} \\
(\xi) A_{=}^{-1}(\xi) \tilde{l}(\xi)++A_{\neq}^{-1}(\xi) V_{-a} F\left(\sum_{k=1}^{n} c_{k}\left(x^{\prime}\right) \delta^{(k-1)}\left(x_{m}\right)\right) .
\end{gathered}
$$

The a priori estimate can be proved by a usual method. ${ }^{1,3}$

To refine the formula (5), we write

$$
\begin{aligned}
(F T u)(\xi) & =\int_{\mathbf{R}^{m}} e^{i x \cdot \xi^{\prime}} u\left(x_{1}, \cdots, x_{m-1}, x_{m}-a\left|x^{\prime}\right|\right) d x= \\
& =\int_{\mathbf{R}^{m}} e^{i y^{\prime} \xi^{\prime}} e^{i\left(y_{m}+a\left|y^{\prime}\right|\right) \xi_{m}} u\left(y_{1}, \cdots, y_{m-1}, y_{m}\right) d y=\int_{\mathbf{R}^{m-1}} e^{i a\left|y^{\prime}\right| \xi_{m}} e^{i y^{\prime} \xi^{\prime}} \hat{u}\left(y_{1}, \cdots, y_{m-1}, \xi_{m}\right) d y^{\prime},
\end{aligned}
$$

where $\hat{u}$ denotes the Fourier transform on the last variable, and Jacobian is 


$$
\frac{D\left(x_{1}, x_{2}, \cdots, x_{m}\right)}{D\left(y_{1}, y_{2}, \cdots, y_{m}\right)}=\left|\begin{array}{cccr}
1 & 0 & \cdots 0 & 0 \\
0 & 1 & \cdots 0 & 0 \\
0 & 0 & \cdots 1 & 0 \\
& \cdots \cdots \cdots \cdots \cdots & & \\
-a \operatorname{sign}\left(y_{1}\right) & -a \operatorname{sign}\left(y_{2}\right) & \ldots-a \operatorname{sign}\left(y_{m-1}\right) & 1
\end{array}\right|=1 .
$$

If we define a pseudodifferential operator by the formula

$$
(A u)(x)=\int_{\mathbf{R}^{m}} e^{-i x \xi} A(\xi) \tilde{u}(\xi) d \xi
$$

and the direct Fourier transform

$$
\tilde{u}(\xi)=\int_{\mathbf{R}^{m}} e^{i x \xi} u(x) d x
$$

then we have the following relation formally at least

$$
(F T u)(\xi)=\int_{\mathbf{R}^{m-1}} e^{i\left(a_{1}\left|y_{1}\right|+\ldots+a_{m-1}\left|y_{m-1}\right|\right) \xi_{m}} e^{i y^{\prime} \xi^{\prime}} \hat{u}\left(y_{1}, \ldots, y_{m-1}, \xi_{m}\right) d y^{\prime}
$$

In other words, if we will denote the $(m-1)$-dimensional Fourier transform ( $y^{\prime} \rightarrow \xi^{\prime}$ in distribution sense) of function $e^{i\left(a_{1}\left|y_{1}\right|+\ldots+a_{m-1}\left|y_{m-1}\right|\right) \xi_{m}}$ by $E_{a}\left(\xi^{\prime}, \xi_{m}\right)$, then the formula (7) will be the following:

$$
(F T u)(\xi)=\left(E_{a} * \tilde{u}\right)(\xi),
$$

where the sign $*$ denotes a convolution for the first $m-1$ variables and the multiplier for the last variable $\xi_{m}$. Thus, $V_{\mathbf{a}}$ is a combination of a convolution operator and the multiplier with the kernel $E_{a}\left(\xi^{\prime}, \xi_{m}\right)$. It is a very good operator, and it is bounded in Sobolev-Slobodetskii spaces $H^{s}\left(\mathbf{R}^{m}\right)$.

\section{5 | POLYSINGULAR INTEGRALS AND POTENTIALS}

Here, we consider the formula (7) more precisely, and we will try to obtain an explicit expression for this Fourier transform. Obviously,

$$
\begin{aligned}
& \int_{\mathbf{R}^{m-1}} e^{i\left(a_{1}\left|y_{1}\right|+\ldots+a_{m-1}\left|y_{m-1}\right|\right) \xi_{m}} e^{i y^{\prime} \xi^{\prime}} \hat{u}\left(y_{1}, \ldots, y_{m-1}, \xi_{m}\right) d y^{\prime}= \\
& \int_{-\infty}^{+\infty} e^{i\left(a_{1}\left|y_{1}\right| \xi_{m}+y_{1} \xi_{1}\right)} \ldots \int_{-\infty}^{+\infty} e^{i\left(a_{m-1}\left|y_{m-1}\right| \xi_{m}+y_{m-1} \xi_{m-1}\right)} \hat{u}\left(y_{1}, \ldots, y_{m-1}, \xi_{m}\right) d y^{\prime},
\end{aligned}
$$

and to move further, we need to study the following one-dimensional integral 


$$
\begin{aligned}
(S f)\left(\xi_{k}, \xi_{m}\right) & =\int_{-\infty}^{+\infty} e^{i\left(a_{k}|y| \xi_{m}+y \xi_{k}\right)} f(y) d y \\
& =\int_{-\infty}^{0} e^{i y\left(\xi_{k}-a_{k} \xi_{m}\right)} f(y) d y+\int_{0}^{+\infty} e^{i y\left(\xi_{k}+a_{k} \xi_{m}\right)} f(y) d y \\
& =\int_{-\infty}^{+\infty} e^{i y\left(\xi_{k}-a_{k} \xi_{m}\right)} \chi_{-}(y) f(y) d y+\int_{-\infty}^{+\infty} e^{i y\left(\xi_{k}+a_{k} \xi_{m}\right)} \chi_{+}(y) f(y) d y
\end{aligned}
$$

where $\chi_{ \pm}$is an indicator of $\mathbb{R}_{ \pm}$.

According to Eskin, ${ }^{3}$ we have the following relations:

$$
\begin{gathered}
\int_{-\infty}^{+\infty} e^{i y\left(\xi_{k}-a_{k} \xi_{m}\right)} \chi_{-}(y) f(y) d y=\frac{1}{2} \tilde{f}\left(\xi_{k}-a_{k} \xi_{m}\right)-v \cdot p \cdot \frac{i}{2 \pi} \int_{-\infty}^{+\infty} \frac{\tilde{f}(\eta) d \eta}{\xi_{k}-a_{k} \xi_{m}-\eta} \\
\int_{-\infty}^{+\infty} e^{i y\left(\xi_{k}+a_{k} \xi_{m}\right)} \chi_{+}(y) f(y) d y=\frac{1}{2} \tilde{f}\left(\xi_{k}+a_{k} \xi_{m}\right)+v \cdot p \cdot \frac{i}{2 \pi} \int_{-\infty}^{+\infty} \frac{\tilde{f}(\eta) d \eta}{\xi_{k}+a_{k} \xi_{m}-\eta}
\end{gathered}
$$

so we have

$$
\begin{aligned}
& \int_{-\infty}^{+\infty} e^{i\left(a_{k}|y| \xi_{m}+y \xi_{k}\right)} f(y) d y=\frac{\tilde{f}\left(\xi_{k}-a_{k} \xi_{m}\right)+\tilde{f}\left(\xi_{k}+a_{k} \xi_{m}\right)}{2}+ \\
& v \cdot p \cdot \frac{i}{2 \pi} \int_{-\infty}^{+\infty} \frac{\tilde{f}(\eta) d \eta}{\xi_{k}+a_{k} \xi_{m}-\eta}-v \cdot p \cdot \frac{i}{2 \pi} \int_{-\infty}^{+\infty} \frac{\tilde{f}(\eta) d \eta}{\xi_{k}-a_{k} \xi_{m}-\eta} .
\end{aligned}
$$

Since we need to do the same on each variable, it seems we will get a very trouble representation. That is why we will restrict them by a three-dimensional case.

\section{6 | THREE-DIMENSIONAL CASE, A PYRAMID, AND BISINGULAR OPERATORS}

Thus, we consider here $m=3$, and for calculating the operator $V_{\mathbf{a}}, \mathbf{a}=\left(a_{1}, a_{2}\right)$, we evaluate

$$
\begin{aligned}
& \int_{\mathbf{R}^{2}} e^{i\left(a_{1}\left|y_{1}\right|+a_{2}\left|y_{2}\right|\right) \xi_{3}} e^{i\left(y_{1} \xi_{1}+y_{2} \xi_{2}\right)} \hat{u}\left(y_{1}, y_{2}, \xi_{3}\right) d y_{1} d y_{2}= \\
& \int_{-\infty}^{+\infty} e^{i\left(a_{1}\left|y_{1}\right| \xi_{3}+y_{1} \xi_{1}\right)}\left(\int_{-\infty}^{+\infty} e^{i\left(a_{2}\left|y_{2}\right| \xi_{3}+y_{2} \xi_{2}\right)} \hat{u}\left(y_{1}, y_{2}, \xi_{3}\right) d y_{2}\right) d y_{1}= \\
& \int_{-\infty}^{+\infty} e^{i\left(a_{1}\left|y_{1}\right| \xi_{3}+y_{1} \xi_{1}\right)}\left(\hat{\hat{u}}\left(y_{1}, \xi_{2}-a_{2} \xi_{3}, \xi_{3}\right)+\frac{\hat{\hat{u}}\left(y_{1}, \xi_{2}+a_{2} \xi_{3}, \xi_{3}\right)}{2}+v \cdot p \cdot \frac{i}{2 \pi} \int_{-\infty}^{+\infty} \frac{\hat{u}\left(y_{1}, \eta, \xi_{3}\right) d \eta}{\xi_{2}+a_{2} \xi_{3}-\eta}-v \cdot p \cdot \frac{i}{2 \pi} \int_{-\infty}^{+\infty} \frac{\hat{u}\left(y_{1}, \eta, \xi_{3}\right) d \eta}{\xi_{2}-a_{2} \xi_{3}-\eta}\right) d y_{1},
\end{aligned}
$$


where $\hat{u}$ denotes the Fourier transform on the last variable $y_{3}$ and $\hat{u}$ denotes the Fourier transform on two last variables $y_{2}, y_{3}$.

Let us denote

$$
\begin{aligned}
v_{1}(\xi)= & \frac{1}{2} \int_{-\infty}^{+\infty} e^{i\left(a_{1}\left|y_{1}\right| \xi_{3}+y_{1} \xi_{1}\right)} \hat{\hat{u}}\left(y_{1}, \xi_{2}-a_{2} \xi_{3}, \xi_{3}\right) d y_{1}, \\
v_{2}(\xi)= & \frac{1}{2} \int_{-\infty}^{+\infty} e^{i\left(a_{1}\left|y_{1}\right| \xi_{3}+y_{1} \xi_{1}\right)} \hat{\hat{u}}\left(y_{1}, \xi_{2}+a_{2} \xi_{3}, \xi_{3}\right) d y_{1}, \\
w_{1}(\xi)= & \int_{-\infty}^{+\infty} e^{i\left(a_{1}\left|y_{1}\right| \xi_{3}+y_{1} \xi_{1}\right)}\left(S_{2} \hat{\hat{u}}\right)\left(y_{1}, \xi_{2}+a_{2} \xi_{3}\right) d y_{1}, \\
w_{2}(\xi)= & \int_{-\infty}^{+\infty} e^{i\left(a_{1}\left|y_{1}\right| \xi_{3}+y_{1} \xi_{1}\right)}\left(S_{2} \hat{\hat{u}}\right)\left(y_{1}, \xi_{2}-a_{2} \xi_{3}\right) d y_{1},
\end{aligned}
$$

where

$$
\left(S_{2} u\right)\left(\xi_{1}, \xi_{2}, \xi_{3}\right)=v \cdot p \frac{i}{2 \pi} \int_{-\infty}^{+\infty} \frac{u\left(\xi_{1}, \eta, \xi_{3}\right) d \eta}{\xi_{2}-\eta}
$$

Further, taking into account (8) and (9), the fact $F_{y_{1} \rightarrow \xi_{1}} \hat{\hat{u}} \equiv \tilde{u}$ and the integral

$$
\left(S_{1} u\right)\left(\xi_{1}, \xi_{2}, \xi_{3}\right)=v \cdot p \frac{i}{2 \pi} \int_{-\infty}^{+\infty} \frac{u\left(\tau, \xi_{2}, \xi_{3}\right) d \tau}{\xi_{1}-\tau}
$$

we obtain

$$
\begin{aligned}
& \int_{\mathbf{R}^{2}} e^{i\left(a_{1}\left|y_{1}\right|+a_{2}\left|y_{2}\right|\right) \xi_{3}} e^{i\left(y_{1} \xi_{1}+y_{2} \xi_{2}\right)} \hat{u}\left(y_{1}, y_{2}, \xi_{3}\right) d y_{1} d y_{2}= \\
& \frac{\tilde{u}\left(\xi_{1}-a_{1} \xi_{3}, \xi_{2}-a_{2} \xi_{3}, \xi_{3}\right)+\tilde{u}\left(\xi_{1}+a_{1} \xi_{3}, \xi_{2}-a_{2} \xi_{3}, \xi_{3}\right)}{4} \\
& +\frac{1}{2}\left(S_{1} \tilde{u}\right)\left(\xi_{1}+a_{1} \xi_{3}, \xi_{2}-a_{2} \xi_{3}, \xi_{3}\right)-\frac{1}{2}\left(S_{1} \tilde{u}\right)\left(\xi_{1}-a_{1} \xi_{3}, \xi_{2}-a_{2} \xi_{3}, \xi_{3}\right) \\
& \quad+\frac{\tilde{u}\left(\xi_{1}-a_{1} \xi_{3}, \xi_{2}+a_{2} \xi_{3}, \xi_{3}\right)+\tilde{u}\left(\xi_{1}+a_{1} \xi_{3}, \xi_{2}+a_{2} \xi_{3}, \xi_{3}\right)}{4} \\
& +\frac{1}{2}\left(S_{1} \tilde{u}\right)\left(\xi_{1}+a_{1} \xi_{3}, \xi_{2}+a_{2} \xi_{3}, \xi_{3}\right)-\frac{1}{2}\left(S_{1} \tilde{u}\right)\left(\xi_{1}-a_{1} \xi_{3}, \xi_{2}+a_{2} \xi_{3}, \xi_{3}\right) \\
& \quad+\frac{\left(S_{2} \tilde{u}\right)\left(\xi_{1}-a_{1} \xi_{3}, \xi_{2}+a_{2} \xi_{3}, \xi_{3}\right)+\left(S_{2} \tilde{u}\right)\left(\xi_{1}+a_{1} \xi_{3}, \xi_{2}+a_{2} \xi_{3}, \xi_{3}\right)}{2} \\
& +\left(S_{1} S_{2} \tilde{u}\right)\left(\xi_{1}+a_{1} \xi_{3}, \xi_{2}+a_{2} \xi_{3}, \xi_{3}\right)-\left(S_{1} S_{2} \tilde{u}\right)\left(\xi_{1}-a_{1} \xi_{3}, \xi_{2}+a_{2} \xi_{3}, \xi_{3}\right) \\
& \quad-\frac{\left(S_{2} \tilde{u}\right)\left(\xi_{1}-a_{1} \xi_{3}, \xi_{2}-a_{2} \xi_{3}, \xi_{3}\right)+\left(S_{2} \tilde{u}\right)\left(\xi_{1}+a_{1} \xi_{3}, \xi_{2}-a_{2} \xi_{3}, \xi_{3}\right)}{2} \\
& -\left(S_{1} S_{2} \tilde{u}\right)\left(\xi_{1}+a_{1} \xi_{3}, \xi_{2}-a_{2} \xi_{3}, \xi_{3}\right)+\left(S_{1} S_{2} \tilde{u}\right)\left(\xi_{1}-a_{1} \xi_{3}, \xi_{2}-a_{2} \xi_{3}, \xi_{3}\right) .
\end{aligned}
$$

Thus, we see that the operator $V_{a}$ is composed from operators $S_{1}, S_{2}$, and a certain change of variables.

Exercise 1. (Exercise 1) Find the representation for the operator $V_{a}$ for a polyhedral angle. 


\section{7 | BOUNDARY VALUE PROBLEMS FOR A POLYHEDRAL ANGLE}

Let us consider a simple boundary value problem for the equation

$$
(A u)(x)=0, \quad x \in C_{+}^{a}
$$

for the case $æ-s=1+\delta,|\delta|<1 / 2$, where $A$ is an elliptic pseudodifferential operator admitting the wave factorization with respect to the cone $C_{+}^{a}$.

According to Theorem 1, we have the formula for a general solution; for our case, it can be written as

$$
\tilde{u}(\xi)=A_{\neq}^{-1}(\xi)\left(V_{-a} F c_{0}\right)(\xi)
$$

where $c_{0}\left(x^{\prime}\right)$ is an arbitrary function from $H^{s_{0}}\left(\mathbb{R}^{2}\right)$.

\section{1 | Transformations of a general solution}

Now we need to choose additional conditions to determine uniquely this function. First, we will do the following: We will write an expression for $V_{-a} F c_{0}$, and then we will see what kind of conditions for a solution $u$ is more preferable. Using our previous calculations, we can write

$$
\begin{aligned}
A_{\neq}(\xi) \tilde{u}(\xi)= & \tilde{C}_{1}\left(\xi_{1}-a_{1} \xi_{3}, \xi_{2}-a_{2} \xi_{3}\right)+\tilde{C}_{2}\left(\xi_{1}-a_{1} \xi_{3}, \xi_{2}+a_{2} \xi_{3}\right) \\
& +\tilde{C}_{3}\left(\xi_{1}+a_{1} \xi_{3}, \xi_{2}-a_{2} \xi_{3}\right)+\tilde{C}_{1}\left(\xi_{1}+a_{1} \xi_{3}, \xi_{2}+a_{2} \xi_{3}\right),
\end{aligned}
$$

where

$$
\begin{aligned}
& \tilde{C}_{1}\left(\xi_{1}-a_{1} \xi_{3}, \xi_{2}-a_{2} \xi_{3}\right)=\frac{1}{4} \tilde{c}_{0}\left(\xi_{1}-a_{1} \xi_{3}, \xi_{2}-a_{2} \xi_{3}\right)-\frac{1}{2}\left(S_{1} \tilde{c}_{0}\right)\left(\xi_{1}-a_{1} \xi_{3}, \xi_{2}-a_{2} \xi_{3}\right)- \\
& \quad-\frac{1}{2}\left(S_{2} \tilde{c}_{0}\right)\left(\xi_{1}-a_{1} \xi_{3}, \xi_{2}-a_{2} \xi_{3}\right)+\left(S_{1} S_{2} \tilde{c}_{0}\right)\left(\xi_{1}-a_{1} \xi_{3}, \xi_{2}-a_{2} \xi_{3}\right) \\
& \tilde{C}_{2}\left(\xi_{1}-a_{1} \xi_{3}, \xi_{2}+a_{2} \xi_{3}\right)=\frac{1}{4} \tilde{c}_{0}\left(\xi_{1}-a_{1} \xi_{3}, \xi_{2}+a_{2} \xi_{3}\right)-\frac{1}{2}\left(S_{1} \tilde{c}_{0}\right)\left(\xi_{1}-a_{1} \xi_{3}, \xi_{2}+a_{2} \xi_{3}\right)+ \\
& \quad+\frac{1}{2}\left(S_{2} \tilde{c}_{0}\right)\left(\xi_{1}-a_{1} \xi_{3}, \xi_{2}+a_{2} \xi_{3}\right)-\left(S_{1} S_{2} \tilde{c}_{0}\right)\left(\xi_{1}-a_{1} \xi_{3}, \xi_{2}+a_{2} \xi_{3}\right) ; \\
& \tilde{C}_{3}\left(\xi_{1}+a_{1} \xi_{3}, \xi_{2}-a_{2} \xi_{3}\right)=\frac{1}{4} \tilde{c}_{0}\left(\xi_{1}+a_{1} \xi_{3}, \xi_{2}-a_{2} \xi_{3}\right)+\frac{1}{2}\left(S_{1} \tilde{c}_{0}\right)\left(\xi_{1}+a_{1} \xi_{3}, \xi_{2}-a_{2} \xi_{3}\right)+ \\
& \quad+\frac{1}{2}\left(S_{2} \tilde{c}_{0}\right)\left(\xi_{1}+a_{1} \xi_{3}, \xi_{2}-a_{2} \xi_{3}\right)-\left(S_{1} S_{2} \tilde{c}_{0}\right)\left(\xi_{1}+a_{1} \xi_{3}, \xi_{2}-a_{2} \xi_{3}\right) ; \\
& \tilde{C}_{4}\left(\xi_{1}+a_{1} \xi_{3}, \xi_{2}+a_{2} \xi_{3}\right)=\frac{1}{4} \tilde{c}_{0}\left(\xi_{1}-a_{1} \xi_{3}, \xi_{2}-a_{2} \xi_{3}\right)+\frac{1}{2}\left(S_{1} \tilde{c}_{0}\right)\left(\xi_{1}+a_{1} \xi_{3}, \xi_{2}+a_{2} \xi_{3}\right)+ \\
& \quad+\frac{1}{2}\left(S_{2} \tilde{c}_{0}\right)\left(\xi_{1}+a_{1} \xi_{3}, \xi_{2}+a_{2} \xi_{3}\right)+\left(S_{1} S_{2} \tilde{c}_{0}\right)\left(\xi_{1}+a_{1} \xi_{3}, \xi_{2}+a_{2} \xi_{3}\right) .
\end{aligned}
$$

It is very interesting that the last formulas are very similar to the well-known formulas for boundary values of a twodimensional Cauchy type integral. ${ }^{16}$

\section{2 | Simplest solvable boundary value problem with a nonlocal condition}

It seems the problem of finding the unknown function $c_{0}\left(\xi_{1}, \xi_{2}\right)$ is very hard, but we suppose that we know the following function $\tilde{u}\left(\xi_{1}, \xi_{2}, 0\right)$. It means that we know the following integral

$$
\int_{-\infty}^{+\infty} u\left(x_{1}, x_{2}, x_{3}\right) d x_{3} \equiv g\left(x_{1}, x_{2}\right) \text {; }
$$

thus, 


$$
\tilde{u}\left(\xi_{1}, \xi_{2}, 0\right)=\tilde{g}\left(\xi_{1}, \xi_{2}\right)
$$

\section{3 | Studying a general solution and lifting}

The formula (11) includes a representation for $V_{-a} \tilde{c}_{0}$, where $\tilde{c}_{0}\left(\xi^{\prime}\right)$ is a function of two variables. Thus, if $\tilde{c}_{0}\left(\xi_{1}, \xi_{2}\right)$ depends on two variables $\xi_{1}, \xi_{2}$, then $V_{-a} \tilde{c}_{0}$ depends on all three variables $\xi_{1}, \xi_{2}, \xi_{3}$.

Lemma 1. If $d\left(x_{1}, x_{2}\right) \in H^{s}\left(\mathbf{R}^{2}\right)$, then $\mathrm{D}\left(\mathrm{x}_{1}, \mathrm{x}_{2}, \mathrm{x}_{3}\right)=\mathrm{d}\left(\mathrm{x}_{1}+\mathrm{a}_{1} \mathrm{x}_{3}, \mathrm{x}_{2}+\mathrm{a}_{2} \mathrm{x}_{3}\right)$ belongs to the space $\mathrm{H}^{\mathrm{s}-1 / 2}\left(\boldsymbol{R}^{3}\right)$.

Proof Let us estimate

$$
\tilde{D}\left(\xi_{1}, \xi_{2}, \xi_{3}\right)=\int_{R^{3}} e^{i x \cdot \xi} D(x) d x=\int_{-\infty}^{+\infty}\left(\int_{R^{2}} e^{i\left(x_{1} \xi_{1}+x_{2} \xi_{2}\right)} d\left(x_{1}+a_{1} x_{3}, x_{2}+a_{2} x_{3}\right) d x_{1} d x_{2}\right) e^{i x_{3} \xi_{3}} d x_{3} .
$$

Let us consider the inner two-dimensional integral

$$
\int_{R^{2}} e^{i\left(x_{1} \xi_{1}+x_{2} \xi_{2}\right)} d\left(x_{1}+a_{1} x_{3}, x_{2}+a_{2} x_{3}\right) d x_{1} d x_{2}
$$

and apply the change of variables

$$
\begin{aligned}
& x_{1}+a_{1} x_{3}=t_{1} \\
& x_{2}+a_{2} x_{3}=t_{2}
\end{aligned}
$$

and

$$
x_{1} \xi_{1}+x_{2} \xi_{2}=\left(t_{1}-a_{1} x_{3}\right) \xi_{1}+\left(t_{2}-a_{2} x_{3}\right) \xi_{2}=t_{1} \xi_{1}+t_{2} \xi_{2}-\left(a_{1} \xi_{1}+a_{2} \xi_{2}\right) x_{3}
$$

and we have

$$
\tilde{D}\left(\xi_{1}, \xi_{2}, \xi_{3}\right)=\tilde{d}\left(\xi_{1}, \xi_{2}\right) \int_{-\infty}^{+\infty} e^{i x_{3}\left(\xi_{3}-a_{1} \xi_{1}-a_{2} \xi_{2}\right)} d x_{3}=\tilde{d}\left(\xi_{1}, \xi_{2}\right) \delta\left(\xi_{3}-a_{1} \xi_{1}-a_{2} \xi_{2}\right)
$$

Therefore,

$$
\|D\|_{s}^{2}=\int_{R^{3}}(1+|\xi|)^{2 s}|\tilde{D}(\xi)|^{2} d \xi \sim \int_{\Gamma} \tilde{d}\left(\xi_{1}, \xi_{2}\right)\left(\int_{-\infty}^{+\infty}\left(1+\left|\xi^{\prime}\right|+\left|\xi_{3}\right|\right)^{2 s} d \xi_{3}\right) d \xi^{\prime}
$$

where $\Gamma$ is the hyperplane $\xi_{3}-a_{1} \xi_{1}-a_{2} \xi_{2}=0$ in $\mathbf{R}^{3}$.

Further, we take $\beta \in \mathbf{R}$ so that $\beta>1$ and taking into account that $\left(1+\left|\xi^{\prime}\right|+\left|\xi_{3}\right|\right)^{2 s+\beta} \sim\left(1+\left|\xi^{\prime}\right|\right)^{2 s+\beta}$ on $\Gamma$ and

$$
\left(1+\mid\left(\xi^{\prime}|+| \xi_{3} \mid\right)^{2 s}=\frac{\left(1+\mid\left(\xi^{\prime}|+| \xi_{3} \mid\right)^{2 s+\beta}\right.}{\left(1+\left|\xi^{\prime}\right|+\left|\xi_{3}\right|\right)^{\beta}}\right.
$$

after integrating, we have

$$
\int_{-\infty}^{+\infty}\left(1+\left|\xi^{\prime}\right|+\left|\xi_{3}\right|\right)^{-\beta} d \xi_{3}=2 \int_{0}^{+\infty}\left(1+\left|\xi^{\prime}\right|+\xi_{3}\right)^{-\beta} d \xi_{3} \sim\left(1+\left|\xi^{\prime}\right|\right)^{-\beta+1}
$$

and it implies 


$$
\|D\|_{s} \sim \int R^{2}\left|\tilde{d}\left(\xi^{\prime}\right)\right|^{2}\left(1+\left|\xi^{\prime}\right|\right)^{2 s+1} d \xi^{\prime}=[d]_{S+1 / 2}
$$

\section{4 | One-dimensional singular integral equation}

Substituting (14) into (12) and collecting similar summands, we obtain the following equation for the unknown $\tilde{c}_{0}\left(\xi^{\prime}\right)$

$$
A_{\neq}^{-1}\left(\xi^{\prime}, 0\right)\left(\tilde{c}_{0}\left(\xi^{\prime}\right)+\left(S_{2} \tilde{c}_{0}\right)\left(\xi^{\prime}\right)\right)=\tilde{g}\left(\xi^{\prime}\right)
$$

or if we designate $A_{\neq}\left(\xi^{\prime}, 0\right) \tilde{g}\left(\xi^{\prime}\right) \equiv f\left(\xi^{\prime}\right)$

$$
\tilde{c}_{0}\left(\xi^{\prime}\right)+\left(S_{2} \tilde{c}_{0}\right)\left(\xi^{\prime}\right)=\tilde{f}\left(\xi^{\prime}\right)
$$

Equation 15 is a one-dimensional singular integral equation with a parameter $\xi_{1}$. It can be solved (if it is possible) by standard methods. ${ }^{16}$ Indeed, since $S_{2}^{2}=-1 / 2 I$, we easily obtain acting by $S_{2}$ for (15)

$$
\left(S_{2} \tilde{c}_{0}\right)\left(\xi^{\prime}\right)-1 / 2 \tilde{c}_{0}\left(\xi^{\prime}\right)=\left(S_{2} \tilde{f}\right)\left(\xi^{\prime}\right),
$$

from which by subtraction, we have

$$
\tilde{c}_{0}\left(\xi^{\prime}\right)=2 / 3\left(\tilde{f}\left(\xi^{\prime}\right)-\left(S_{2} \tilde{f}\right)\left(\xi^{\prime}\right)\right)
$$

Now if we have found $\tilde{c}_{0}\left(\xi^{\prime}\right)$, we have the solution of the problem (10) and (13).

\section{5 | A priori estimates}

Here, we will give the a priori estimates for the solution. Since

$$
\tilde{u}(\xi)=A_{\neq}^{-1}(\xi)\left(V_{-a} \tilde{c}_{0}\right)(\xi),
$$

Theorem 2. Let $\mathrm{A}(\xi)$ admits the wave factorization with respect to the $C_{+}^{a}$. Then the boundary value problem (10) and (13) has a unique solution for an arbitrary $\mathrm{g} \in \mathrm{H}^{\mathrm{s}+1 / 2}\left(\boldsymbol{R}^{2}\right)$ in the space $H^{s}\left(C_{+}^{a}\right)$. This solution can be constructed explicitly by the Fourier transform and the one-dimensional singular integral operator. The a priori estimate

$$
\|u\|_{s} \leq c[g]_{s+1 / 2}
$$

holds for $-1 / 2<\delta<0$.

Proof. We need to prove the a priori estimate only. Since $f\left(\xi^{\prime}\right)=A_{\neq}\left(\xi^{\prime}, 0\right) \tilde{g}\left(\xi^{\prime}\right)$, then $A_{\neq}\left(\xi^{\prime}, 0\right) \tilde{g}\left(\xi^{\prime}\right) \in \tilde{H}^{s+1 / 2-\infty}\left(\mathbf{R}^{2}\right)=H^{s_{0}}\left(\mathbf{R}^{2}\right)$. We remind

$$
s_{0}=s-\propto+1 / 2=-1 / 2-\delta,
$$

so that $-1 / 2<s_{0}<0$. Then we have according to (16)

$$
\left[\tilde{c}_{0}\right]_{S_{0}} \leq c\left([\tilde{f}]_{S_{0}}+\left[S_{2} \tilde{f}\right]_{S_{0}}\right) \leq c[\tilde{f}]_{S_{0}}
$$

because the operator $S_{2}$ is bounded in $\tilde{H}^{s_{0}}\left(\mathbf{R}^{2}\right) .{ }^{3}$ Finally,

$$
[\tilde{f}]_{S_{0}} \leq c[\tilde{g}]_{S+1 / 2}
$$

according to properties of pseudodifferential operators. Further, we can use the estimate from Theorem 1. 


\section{8 | CONCLUSION}

We have considered here simplest variant of a boundary value problem, but we hope that the obtained representation for a general solution of the elliptic pseudodifferential equation will help us in considering other boundary value problems like, for example, the Dirichlet and Neumann problems at least. ${ }^{17,18}$ Moreover, we will try to extend our considerations to more complicated singularities, ie, so called thin singularities. ${ }^{19-21}$

\section{ACKNOWLEDGEMENT}

The author is very grateful to referees for their timely and helpful comments.

\section{ORCID}

Vladimir B. Vasilyev (D) http://orcid.org/0000-0001-9351-8084

\section{REFERENCES}

1. Vasil'ev VB. Wave Factorization of Elliptic Symbols: Theory and Applications. Dordrecht-Boston-London: Kluwer Academic Publishers; 2000.

2. Milkhin SG, Prödorf S. Singular Integral Operators. Berlin: Akademie-Verlag; 1986.

3. Eskin GI. Boundary Value Problems for Elliptic Pseudodifferential Equations. Providence: AMS; 1981.

4. Simonenko IB. Local method in the theory of translation invariant operators and their envelopes. Rostov on Don, CVVR. (Russian); 2007.

5. Nazarov SA, Plamenevsky BA. Elliptic Problems in Domains with Piecewise Smooth Boundaries. Berlin-New York: Walter de Gruyter; 1994.

6. Schulze B-W, Sternin B, Shatalov V. Differential Equations on Singular Manifolds: Semiclassical Theory and Operator Algebras. Berlin: Wiley-VCH; 1998.

7. Nazaikinskii VE, Savin AYU, Schulze B-W, Sternin B. Elliptic Theory on Singular Manifolds. Boca Raton: Chapman \& Hall/CRC; 2006.

8. Nazaikinskii V, Schulze B-W, Sternin B. The Localization Problem in Index Theory of Elliptic Operators. Pseudo-Differential Operators Theory and Applications, Vol. 10. Basel: Birkhäuser/Springer; 2014.

9. Karlovich YI, Rodino LG, Silbermann B, eds. Operator Theory, Pseudo-Differential Equations, and Mathematical Physics. Basel: Birkhäuser Springer; 2013.

10. Kottke C. Generalized blow-up of corners and fiber products. Trans Am Math Soc. 2015;367(1):651-705.

11. Vasilyev VB. Pseudo differential equations on manifolds with non-smooth boundaries. In: Pinelas S, Chipot M, Došlá Z, eds. Differential and Difference Equations and Applications, V. 47. Springer Proc. Math. \& Stat.; 2013:625-637.

12. Vasilyev VB. Multipliers of Fourier Integrals, Pseudodifferential Equations, the Wave Factorization, Boundary Value Problems,. 2nd ed. Moscow: Editorial URSS; 2010. (Russian).

13. Cordero E, Feichtinger H, Luef F. Banach Gelfand triples for Gabor analysis. In: Rodino L, WongMW, eds. Pseudo-Differential Operators: Quantization and Signals, Lect. Notes Math., vol. 1949. Berlin: Springer; 2008:1-33.

14. Rodino L, Schulze B-W, Wong MW, eds. Pseudo-Differential Operators: Partial Differential Equations and Time-Frequency Analysis (Fields Institute Communications). Providence, AMS: American Mathematical Soc; 2007.

15. Vladimirov VS. Generalized Functions in Mathematical Physics. Moscow: Mir; 1979.

16. Gakhov FD. Boundary Value Problems. NY: Dover Publications; 1981.

17. Vasilyev V. On certain elliptic problems for pseudo differential equations in a polyhedral cone. Adv Dyn Syst Appl. 2014;9(2):227-237.

18. Vasilyev VB. On the Dirichlet and Neumann problems in multi-dimensional cone. Math Bohem. 2014;139(2):333-340.

19. Vasilyev VB. Potentials for elliptic boundary value problems in cones. Siberian Electron Math Repts. 2016;13:1129-1149. (Russian).

20. Vasilyev VB. Asymptotical analysis of singularities for pseudo differential equations in canonical non-smooth domains. In: Constanda C, Harris PJ, eds. Integral Methods in Science and Engineering. Computational and Analytic Aspects. Boston: Birkhäuser; 2011:379-390.

21. Vasilyev VB. New Constructions in the Theory of Elliptic Boundary Value Problems. In: Integral Methods in Science and Engineering. Proc. IMSE Conference, Karlsruhe, Germany, 2014. Birkhäuser: Basel; 2015:629-641.

How to cite this article: Vasilyev VB. Pseudo-differential equations, wave factorization, and related problems. Math Meth Appl Sci. 2018;41:9252-9263. https://doi.org/10.1002/mma.5212 